\title{
Martingale theory for housekeeping heat
}

\author{
RaphaËl ChÉtrite ${ }^{1}$, Shamik Gupta ${ }^{2}$, IzaAk Neri ${ }^{3,4}$ and Édgar Roldán ${ }^{5}$ \\ 1 Université Côte d'Azur, CNRS, LJAD, Parc Valrose, 06108 Nice Cedex 02, France \\ 2 Department of Physics, Ramakrishna Mission Vivekananda University, Belur Math, Howrah 711 202, India \\ 3 Department of Mathematics, King's College London, Strand, London WC2R 2LS, United Kingdom \\ ${ }^{4}$ Max Planck Institute for the Physics of Complex Systems, Nöthnitzer Strasse 38, 01187 Dresden, Germany \\ ${ }_{5}$ ICTP - The Abdus Salam International Centre for Theoretical Physics, Strada Costiera 11, 34151 Trieste, Italy
}

PACS 05.70.Ln - Nonequilibrium and irreversible thermodynamics PACS 05.40. Ca - Noise

\begin{abstract}
The housekeeping heat is the energy exchanged between a system and its environment in a nonequilibrium process that results from the violation of detailed balance. We describe fluctuations of the housekeeping heat in mesoscopic systems using the theory of martingales, a mathematical framework widely used in probability theory and finance. We show that the exponentiated housekeeping heat (in units of $k_{\mathrm{B}} T$, with $k_{B}$ the Boltzmann constant and $T$ the temperature) of a Markovian nonequilibrium process under arbitrary time-dependent driving is a martingale process. From this result, we derive universal equalities and inequalities for the statistics of stopping-times and suprema of the housekeeping heat. We test our results with numerical simulations of a system driven out of equilibrium and described by Langevin dynamics.
\end{abstract}

Introduction and main results. - A real-valued stochastic process $M_{t}$ is a martingale $1-4$ if it satisfies the following two conditions: (i) the expected value of $M_{t}$ conditioned on its past history $M_{[0, \tau]}$ is

$$
\left\langle M_{t} \mid M_{[0, \tau]}\right\rangle=M_{\tau}
$$

for any time $\tau \leq t$, and (ii) the process is integrable, $\left\langle\left|M_{t}\right|\right\rangle<\infty$ at all times $t$. We use the notation $\langle X \mid Y\rangle \equiv$ $\int X P(X \mid Y) \mathrm{d} X$ for the conditional expectation of $X$ given $Y$, and we write $P(X \mid Y)$ for the conditional probability density of $X$ given $Y$.

We also consider martingales $M_{t}$ relative to a stochastic process $X_{t}$. Such martingale processes satisfy the following three conditions: (i) the expected valu of $M_{t}$ conditioned on the past history $X_{[0, \tau]}$ equals

$$
\left\langle M_{t} \mid X_{[0, \tau]}\right\rangle=M_{\tau}
$$

for any time $\tau \leq t$, (ii) the process is integrable $\left\langle\left|M_{t}\right|\right\rangle<\infty$ at all times $t \geq 0$, and (iii) $M_{t}=f\left(X_{[0, t]}, t\right)$ with $f$ a realvalued function. Note that (2) is a generalization of (1).

Examples of martingales are: (i) the Wiener process $\mathcal{W}_{t}$ (Brownian motion); (ii) a geometric Brownian motion $\Sigma_{t}=e^{\sigma \mathcal{W}_{t}-(1 / 2) \sigma^{2} t}$, where $\sigma$ is a real number; (iii)
Itô processes $I_{t}=\int_{0}^{t} F\left(X_{s}\right) \cdot \mathrm{d} \mathcal{W}_{s}$, where $F(x)$ is a realvalued and well-behaved function 5,6 and $\cdot$ denotes the Itô product; (iv) ratios of probability densities of trajectories $\Lambda_{t}=\mathcal{P}\left(X_{[0, t]}\right) / \mathcal{Q}\left(X_{[0, t]}\right)$ 7] are martingales relative to the process $X_{t}$ if the statistics of the process $X_{t}$ is generated by $\mathcal{Q}$. The average in $(2)$ is then done with respect to the probability $\mathcal{Q}$, i.e., $\left\langle\Lambda_{t} \mid X_{[0, \tau]}\right\rangle_{\mathcal{Q}}=\Lambda_{\tau}$, for all $t \geq \tau$, where $\langle\cdot\rangle_{\mathcal{Q}}$ denotes expectation with respect to $\mathcal{Q}$.

Martingales have found widespread applications in mathematics, economics and gambling 2 2,8 . Gambling strategies can be modelled with stopping times $\mathcal{T}$, which denote the time when a gambler decides to cash out. Stopping times are random times when a stochastic process satisfies for the first time a certain criterion, which is nonanticipative. Examples of stopping times are first-passage times, second-passage times, etc. Stopping times depend on non-anticipative stopping rules i.e. the stopping time $\mathcal{T}$ conditioned on $X_{[0, \mathcal{T}]}$ is independent of the trajectory in the future $X_{(\mathcal{T}, \infty)}$. An important result is Doob's optional stopping theorem [2, which states there exist no winning gambling strategies that can make profit out of a martingale process, irrespective of the stopping rule used:

$$
\left\langle M_{\mathcal{T}}\right\rangle=\left\langle M_{0}\right\rangle,
$$

i.e., the expected value of a martingale at a stopping time $\mathcal{T}$ equals the expected value at the initial time $t=0$. 
Doob's optional stopping theorem (3) holds under some additional conditions on either the stopping time or the martingale process. For example, (3) holds if the stopping time $\mathcal{T}$ satisfies $\mathcal{T} \in[0, \tau]$ with $\tau$ a constant fixed time, or, if $M_{t}$ is uniformly integrable. Another important example for which (3) holds is when $\mathcal{T}$ is a first-passage time with two absorbing thresholds $m_{+}>M_{0}$ and $m_{-}<M_{0}$ and if additionally $\operatorname{Pr}(\mathcal{T}<\infty)=1$, i.e., the probability to quit the game after a finite time is one. We use the latter formulation of the stopping-time theorem in this paper.

In physics, martingales have not been exploited much so far 9 13]. Recent work has applied martingale theory to the thermodynamics of stochastic processes $[9[10]$. In particular, it was found that the exponential of the negative entropy production of a stationary stochastic process is a martingale. Reference [9] uses this observation to derive fluctuation relation for stopping times of entropy production and to derive universal laws on the statistics of infima of the entropy production. This so-called infimum law was tested experimentally in a double electronic dot [14]. Martingales have also been discovered in the context of classical quenched systems 11] and quantum mechanics [12 13. An interesting question is whether there exist other physical processes, in particular, in the context of stochastic energetics, which are martingales.

In this paper, we find a martingale process that describes fluctuations of the heat exchanged between a mesoscopic system and its environment in a nonequilibrium process that takes place at isothermal conditions. Before presenting this martingale process, we review some aspects of nonequilibrium thermodynamics. Following Oono and Paniconi 15], the fluctuating dissipated heat $Q_{t}$ during a single realization of a nonequilibrium process can be decomposed as the sum of two terms, a housekeeping heat $Q_{t}^{\mathrm{hk}}$ and an excess heat $Q_{t}^{\mathrm{ex}} 16,17$. For processes that relax to an equilibrium state, one has $Q_{t}^{\mathrm{hk}}=0$, whereas for nonequilibrium steady states, $\left\langle Q_{t}^{\mathrm{ex}}\right\rangle=0$. Moreover, the housekeeping heat is on average negative, i.e., $\left\langle Q_{t}^{\mathrm{hk}}\right\rangle \leq 0$ for all times $t$; in other words, a stochastic process has on average a tendency to dissipate housekeeping heat. Note that here we use the convention that all heat exchanges are negative when heat flows from the system to its environment. We will also assume that the environment is isothermal at temperature $T$.

Fluctuations of the housekeeping heat also obey universal laws. The housekeeping heat satisfies an integral fluctuation relation: $\left\langle e^{\beta Q_{t}^{\mathrm{hk}}}\right\rangle=1 \quad 17-20$. Here, we use the notation $\beta=\left(k_{\mathrm{B}} T\right)^{-1}$, with $k_{\mathrm{B}}$ the Boltzmann constant. The integral fluctuation relation implies that fluctuations of the housekeeping heat away from the average tendency to dissipate are rare. Indeed, using the Markov inequality on $e^{\beta Q_{t}^{\mathrm{hk}}}$ and the integral fluctuation relation, we find that

$$
\operatorname{Pr}\left(Q_{t}^{\mathrm{hk}} \geq q\right) \leq e^{-\beta q}, \quad q \geq 0 .
$$

Hence, the probability to observe a system that absorbs at least an amount of housekeeping heat $q$ is smaller than $e^{-\beta q}$, so that large fluctuations away from the tendency of a nonequilibrium processes to dissipate occur rarely.

In this paper, we show that the bound (4) on the fluctuations of positive housekeeping heat can be significantly improved. In particular, we show that

$$
\operatorname{Pr}\left(\sup _{\tau \in[0, t]} Q_{\tau}^{\mathrm{hk}} \geq q\right) \leq e^{-\beta q}, \quad q \geq 0,
$$

where $\sup _{\tau \in[0, t]} Q_{\tau}^{\mathrm{hk}}$ is the supremum of the dissipated heat over the full trajectory in the time interval $[0, \tau]$. The bound (5) is tighter than the bound (4), $\operatorname{Pr}\left(Q_{t}^{\text {hk }} \geq q\right) \leq$ $\operatorname{Pr}\left(\sup _{\tau \in[0, t]} Q_{\tau}^{\mathrm{hk}} \geq q\right) \leq e^{-\beta q}$, since the supremum of a trajectory is always larger than its final value, i.e., $\sup _{\tau \in[0, t]} Q_{\tau}^{\mathrm{hk}} \geq Q_{t}^{\mathrm{hk}}$. Remarkably, we show that for continuous stochastic processes, the inequality (5) becomes an equality. Hence, the probability to observe a maximal fluctuation of the housekeeping heat larger than $q$ approaches a time-independent limit equal to $e^{-\beta q}$, which is linked to the fact that $Q_{t}^{\mathrm{hk}}$ has an average tendency to decrease in time. We also derive an integral fluctuation relation at stopping times:

$$
\left\langle e^{\beta Q_{\mathcal{T}}^{\mathrm{hk}}}\right\rangle=1
$$

We remark here that in contrast to the standard fluctuation relation $\left\langle e^{\beta Q_{t}^{\mathrm{hk}}}\right\rangle=1$ 17 20, the average in (6) is over trajectories of different time duration, since $\mathcal{T}$ is a random time. This fluctuation relation $(6)$ holds for a broad class of stopping times $\mathcal{T}$, including first-passage times that are bounded 21 and first-passage times with two absorbing boundaries. Using Jensen's inequality and Eq. (6) gives

$$
\left\langle Q_{\mathcal{T}}^{\mathrm{hk}}\right\rangle \leq 0
$$

which implies that it is not possible to extract housekeeping heat from the thermal reservoir, irrespective of the stopping protocol $\mathcal{T}$ used to end the stochastic process $X_{t}$. In other words, whatever stopping strategy we use it is not possible that on average a system absorbs housekeeping heat from its environment.

The key insight to prove relations (5) is that the process $e^{\beta Q_{t}^{\text {hk }}}$ is a martingale with respect to the physical process $X_{t}$ that dissipates $Q_{t}^{\mathrm{hk}}$, i.e., for any $t \geq \tau$,

$$
\left\langle e^{\beta Q_{t}^{\mathrm{hk}}} \mid X_{[0, \tau]}\right\rangle=e^{\beta Q_{\tau}^{\mathrm{hk}}} .
$$

This result is the cornerstone of this paper, and as we show below it holds for general Markov processes, which include Langevin processes and Markov jump processes. Note that these results share a similarity with those derived in 9 for the stochastic entropy production, with the important distinction that the results here are also valid for nonstationary nonequilibrium processes.

Nonequilibrium heat fluctuations of diffusions. Before discussing general Markov processes, we consider heat fluctuations in $n$-dimensional Itô-diffusion processes of the form

$$
\frac{\mathrm{d} \vec{X}_{t}}{\mathrm{~d} t}=\left[\vec{F}_{t}+\vec{\nabla} \cdot \mathbf{D}_{t}\right]\left(\vec{X}_{t}\right)+\sqrt{2 \mathbf{D}_{t}\left(\vec{X}_{t}\right)} \cdot \vec{\xi}_{t},
$$


where $\vec{F}_{t}$ is a $n$-dimensional vector field and $\mathbf{D}_{t}$ is an invertible diffusion matrix field of dimension $n \times n$. The subindex $t$ denotes an explicit time dependence. The noise $\vec{\xi}_{t}$ is a $n$-dimensional Gaussian white noise with zero mean and covariance matrix equal to the identity matrix. We assume that the Einstein relation holds such that $\vec{F}_{t}=\vec{f}_{t}-\beta \mathbf{D}_{t} \vec{\nabla} U_{t}$ with $\vec{f}_{t}$ an external force and $U_{t}$ a potential. The presence of the term $\vec{\nabla} \cdot \mathbf{D}_{t}$ in $(9)$ ensures that in the case without external force and with time-independent potential, the stationary distribution is the Boltzmann distribution with potential $U$ and inverse temperature $\beta$ 22 24. Note that we could have considered other conventions for the stochastic discretization in (9), e.g., the Fisk-Stratonovich convention 25,26, but the martingality of a process is revealed in a simple mathematical form when using Itô convention. The Fokker-Planck equation associated with the process (9) is

$$
\partial_{t} P_{t}=L_{t}^{\dagger} P_{t},
$$

with

$$
L_{t}=\vec{F}_{t} \cdot \vec{\nabla}+\vec{\nabla} \cdot \mathbf{D}_{t} \cdot \vec{\nabla}
$$

its Markovian generator. In 10, $L_{t}^{\dagger}=-\vec{\nabla} \cdot \vec{F}_{t}+\vec{\nabla}$. $\mathbf{D}_{t} \cdot \vec{\nabla}$ is the formal adjoint of the generator. We define the accompanying distribution 27] $\pi_{t}$ associated to the dynamics as

$$
L_{t}^{\dagger} \pi_{t}=0 .
$$

We remark that $\partial_{t} \pi_{t} \neq L_{t}^{\dagger} \pi_{t}$, i.e., $\pi_{t}$ does not obey the Fokker-Planck equation because the right-hand side of this equation is zero whereas $\partial_{t} \pi_{t} \neq 0$. However, the accompanying distribution would be the stationary distribution for a process with the value of the external parameters constant and equal to those at a given time $s$ (i.e., a process with a generator $L_{t}=L_{s}$ for any $t \geq s$ ). Notice that when the system is driven quasistatically one has $P_{t}=\pi_{t}$.

We now review the stochastic thermodynamics associated with the nonequilibrium fluctuations of Itô processes (9). Such systems possess two type of forces that drives them out of equilibrium: (i) the non-conservative external force $\vec{f}_{t}$, and (ii) the explicit time dependence of the force and diffusion fields. These two sources that drive the system out of equilibrium have distinct thermodynamic fingerprints in terms of heat fluctuations, namely, (i) the fluctuating housekeeping heat 15,17, which for the diffusion process (9) equals

$$
Q_{t}^{\mathrm{hk}} \equiv k_{\mathrm{B}} T \int_{0}^{t}\left[\vec{\nabla} \ln \pi_{s}-\mathbf{D}_{s}^{-1} \cdot \vec{F}_{s}\right]\left(\vec{X}_{s}\right) \circ \mathrm{d} \vec{X}_{s}
$$

and (ii) the fluctuating excess heat 15,17

$$
Q_{t}^{\mathrm{ex}} \equiv-k_{\mathrm{B}} T \int_{0}^{t}\left[\vec{\nabla} \ln \pi_{s}\right]\left(\vec{X}_{s}\right) \circ \mathrm{d} \vec{X}_{s}
$$

where $\circ$ denotes both the use of the Fisk-Stratonovich convention 25 26] and the scalar product. Using Stratonovich calculus, we can write the latter expression as

$$
Q_{t}^{\mathrm{ex}}=-k_{\mathrm{B}} T \ln \frac{\pi_{t}\left(\vec{X}_{t}\right)}{\pi_{0}\left(\vec{X}_{0}\right)}+k_{\mathrm{B}} T \int_{0}^{t} \mathrm{~d} s\left[\partial_{s} \ln \pi_{s}\right]\left(\vec{X}_{s}\right) .
$$

Summing 13 and 14 , we find that the total fluctuating heat associated with a stochastic trajectory [16,

$$
Q_{t} \equiv Q_{t}^{\mathrm{hk}}+Q_{t}^{\mathrm{ex}},
$$

is equal to Sekimoto's expression 16

$$
Q_{t}=-k_{\mathrm{B}} T \int_{0}^{t} \vec{F}_{s}\left(\vec{X}_{s}\right) \cdot \mathbf{D}_{s}^{-1}\left(\vec{X}_{s}\right) \circ \mathrm{d} \vec{X}_{s} .
$$

Equations (12) and (13) imply that the housekeeping heat vanishes in the absence of external forces, i.e., when $\vec{f}_{t}=\overrightarrow{0}$. Nevertheless, heat is dissipated through the excess heat which becomes a border term $Q_{t}^{\mathrm{ex}}=-k_{\mathrm{B}} T \ln \left(\pi_{t}\left(X_{t}\right) / \pi_{0}\left(X_{0}\right)\right)$, see (15). Therefore, the (asymptotic) rate of $Q_{t}^{\text {ex }}$ vanishes when there is no explicit time dependence in the dynamics. In a steady state process without explicit time dependence in the dynamics the housekeeping heat is non-zero, and this is the reason behind the name "housekeeping". An analogous formulation in terms of adiabatic and non-adiabatic entropy production can be found in, e.g., Refs. 1920 .

Martingale features of housekeeping heat for general Markov processes. - In this paragraph we derive generic expression for the housekeeping heat of a Markov process, which is equal to 13 for Itô-diffusion processes. This generic expression allows us to derive universal fluctuation properties of housekeeping heat.

For a Markov process, the housekeeping heat associated with a trajectory $X_{[0, t]}$ of a general Markov process can be written as 2830

$$
Q_{t}^{\mathrm{hk}}=k_{\mathrm{B}} T \ln \frac{\mathcal{P}^{\star}\left[X_{[0, t]}\right]}{\mathcal{P}\left[X_{[0, t]}\right]} .
$$

Here $\mathcal{P}\left[X_{[0, t]}\right]$ is the probability density of a trajectory $X_{[0, t]}$ with initial condition $\rho_{0}$ and the original dynamics given by $L_{t}$. On the other hand, $\mathcal{P}^{\star}\left[X_{[0, t]}\right]$ is the probability density of the same path $X_{[0, t]}$ with the same initial condition $\rho_{0}$ but under a different " $\star$ dynamics" (often called $\pi$-dual dynamics) given by

$$
L_{t}^{\star}=\pi_{t}^{-1} L_{t}^{\dagger} \pi_{t} .
$$

Notably, for general Markov processes, $Q_{t}^{\mathrm{hk}}=0$ for all $t$ when the instantaneous detailed balance condition $\pi_{t}^{-1} L_{t}^{\dagger} \pi_{t}=L_{t}$ is satisfied and thus $\mathcal{P}^{\star}=\mathcal{P}$. This clarifies the adjective "housekeeping" from the fact that it corresponds to the heat absorbed by the system as a result of the violation of instantaneous detailed balance.

Due to the fact that $e^{\beta Q_{t}^{\text {hk }}}=\mathcal{P}^{\star}\left(X_{[0, t]}\right) / \mathcal{P}\left(X_{[0, t]}\right)$ is a path-probability ratio, we obtain directly the cornerstone of this work: for general Markov nonequilibrium processes, 
the exponentiated housekeeping heat in $k_{\mathrm{B}} T$ units is a martingale relative to $X_{0}^{t}$,

$$
\left\langle e^{\beta Q_{t}^{\mathrm{hk}}} \mid X_{[0, \tau]}\right\rangle=e^{\beta Q_{\tau}^{\mathrm{hk}}}
$$

for any $t \geq \tau$. Note that the average here is done with respect to $\mathcal{P}$. Notably, for $\tau=0, X_{[0, \tau]}=X_{0}$, and $(20)$ implies the integral fluctuation relation $\left.\left\langle e^{\beta Q_{t}^{\mathrm{hk}}}\right\rangle=1\right]$. We remark that this property holds for generic Markov processes, which include diffusion processes 29 but also jump processes 28, 31.

The martingality of $e^{\beta Q_{t}^{\text {hk }}}$ entails second-law-like relations for the housekeeping heat. From convexity of $-\ln$, we find that the housekeeping heat is a supermartingale for general Markov nonequilibrium processes. Its expected value in the future $t \geq \tau$ is smaller than or equal to its value at time $\tau$ :

$$
\left\langle Q_{t}^{\mathrm{hk}} \mid X_{[0, \tau]}\right\rangle \leq Q_{\tau}^{\mathrm{hk}}
$$

Note that for $\tau=0$, 21 implies that $\left\langle Q_{t}^{\mathrm{hk}}\right\rangle \leq 0$, as expected.

We now show that 18 is consistent with expression 113 for the housekeeping heat in Itô diffusions. Note that in the particular case of Itô diffusions, given by (9), the "^ dynamics" generates a stochastic process $\vec{Y}_{t}$ described by the Langevin equation

$$
\frac{\mathrm{d} \vec{Y}_{t}}{\mathrm{~d} t}=\left[-\vec{F}_{t}+2 \mathbf{D}_{t} \cdot \vec{\nabla} \ln \pi_{t}+\vec{\nabla} \cdot \mathbf{D}_{t}\right]\left(\vec{Y}_{t}\right)+\sqrt{2 \mathbf{D}_{t}\left(\vec{Y}_{t}\right)} \cdot \vec{\xi}_{t} .
$$

We use the Itô convention, as in (9), to express the housekeeping heat in (13):

$$
\begin{aligned}
Q_{t}^{\mathrm{hk}} & =k_{\mathrm{B}} T \int_{0}^{t}\left[\vec{\nabla} \ln \pi_{s}-\mathbf{D}_{s}^{-1} \cdot \vec{F}_{s}\right]\left(\vec{X}_{s}\right) \cdot \mathrm{d} \vec{X}_{s} \\
& +k_{\mathrm{B}} T \int_{0}^{t} \mathrm{~d} s\left[\mathbf{D}_{s} \cdot \vec{\nabla} \cdot\left(\vec{\nabla} \ln \pi_{s}-\mathbf{D}_{s}^{-1} \cdot \vec{F}_{s}\right)\right]\left(\vec{X}_{s}\right)
\end{aligned}
$$

From (23), we prove in the appendix that the exponentiated housekeeping heat satisfies the equation

$$
\frac{\mathrm{d}}{\mathrm{d} t} e^{\beta Q_{t}^{\mathrm{hk}}}=-\sqrt{2} e^{\beta Q_{t}^{\mathrm{hk}}}\left[\frac{\vec{J}_{t}^{\pi} \cdot \mathbf{D}_{t}^{-1 / 2}}{\pi_{t}}\right]\left(\vec{X}_{t}\right) \cdot \vec{\xi}_{t} .
$$

Here, $\vec{J}_{t}^{\pi} \equiv \vec{F}_{t} \pi_{t}-\mathbf{D}_{t} \cdot \vec{\nabla} \pi_{t}$ is the effective current associated to $\pi_{t}$, and $\vec{\xi}_{t}$ is the same Gaussian white noise as in (9). Equation (24) reveals explicitly the martingale structure of $e^{\beta Q_{t}^{\mathrm{hk}}}$ : due to the absence of drift terms on the right-hand side of 24$), e^{\beta Q_{t}^{\mathrm{hk}}}$ is an Itô process and therefore a martingale. An analogous equation for the exponentiated negative entropy production in nonequilibrium steady states was reported in Ref. [32]. Equations of the type (24) are also used in finance to model pricing options with stochastic volatility 33].
For diffusion processes, the supermartingality of $Q_{t}^{\mathrm{hk}}$ is revealed by using Itô's lemma in (24), leading to 32, 34

$$
\beta \frac{\mathrm{d} Q_{t}^{\mathrm{hk}}}{\mathrm{d} t}=-v^{\mathrm{hk}}\left(\vec{X}_{t}\right)-\sqrt{2 v^{\mathrm{hk}}\left(\vec{X}_{t}\right)} \cdot \xi_{t}^{\mathrm{hk}}\left(\vec{X}_{t}\right),
$$

with

$$
v^{\mathrm{hk}}(\vec{X}) \equiv\left[\frac{\overrightarrow{J_{t}^{\pi}} \cdot \mathbf{D}_{t}^{-1} \cdot \vec{J}_{t}^{\pi}}{\pi_{t}^{2}}\right](\vec{X})
$$

the "entropic drift" 32 of the housekeeping heat, and

$$
\xi_{t}^{\mathrm{hk}}(\vec{X}) \equiv\left[\frac{\mathbf{D}_{t}^{-2} \cdot \vec{J}_{t}^{\pi}}{\sqrt{\vec{J}_{t}^{\pi} \cdot \mathbf{D}_{t}^{-1} \cdot \vec{J}_{t}^{\pi}}} \cdot \overrightarrow{\xi_{t}}\right](\vec{X})
$$

a one-dimensional Gaussian white noise. We remark here that the evolution of $Q_{t}^{\mathrm{hk}}$ given by $(25)$ is driven by the dynamics of $\vec{X}_{t}$, which is autonomous and described by $(9)$. Since $v^{\mathrm{hk}}(\vec{X}) \geq 0$ for all values of $\vec{X}, \sqrt{25}$ reveals that the housekeeping heat is a supermartingale.

Universal stopping and extreme-value statistics from the martingale $e^{\beta Q_{t}^{\text {hk }}}$. - We now discuss the physical implications of the martingale property of $e^{\beta Q_{\mathrm{hk}}}$ for the thermodynamics of nonequilibrium processes. First, we apply Doob's optional sampling theorem (3) to the martingale process $e^{\beta Q_{t}^{\text {hk }}}$, and find an integral fluctuation relation at stopping times 9

$$
\left\langle e^{\beta Q_{\mathcal{T}}^{\mathrm{hk}}}\right\rangle=1
$$

Applying Jensen's inequality $e^{\beta\left\langle Q_{\mathcal{T}}^{\text {hk }}\right\rangle} \leq\left\langle e^{\beta Q_{\mathcal{T}}^{\text {hk }}}\right\rangle=1$ we obtain $\left\langle Q_{\mathcal{T}}^{\text {hk }}\right\rangle \leq 0$. Thus, a nonequilibrium system cannot on average absorb housekeeping heat from its environment by applying stopping rules based on measurements of $Q_{t}^{\mathrm{hk}}$. The relation (28) holds whenever the Doob's optional sampling theorem holds, for example, when $\mathcal{T}$ is a bounded stopping time 21] or when $e^{\beta Q_{t}^{\mathrm{hk}}}$ is uniformly integrable.

The relation (28) also holds for two-boundary firstpassage times $\mathcal{T}_{\mathrm{FP}}$ with thresholds $-q_{-}<0$ or $q_{+}>0$ : $\mathcal{T}_{\mathrm{FP}}$ is the first-time when $Q_{t}^{\mathrm{hk}}$ reaches either $-q_{-}<0$ or $q_{+}>0$. Eq. (28) implies then

$$
\left\langle e^{\beta Q_{\mathcal{T}_{\mathrm{FP}}}^{\mathrm{hk}}}\right\rangle=1
$$

Relation 290 allows us to derive several universal results about the fluctuations of $Q_{t}^{\mathrm{hk}}$.

A process that does not satisfy detailed balance dissipates heat and therefore

$$
\operatorname{Pr}\left(\mathcal{T}_{\mathrm{FP}}<\infty\right)=\mathrm{P}_{-}+\mathrm{P}_{+}=1
$$

with

$$
\mathrm{P}_{-} \equiv \operatorname{Pr}\left[Q_{\mathcal{T}_{\mathrm{FP}}}^{\mathrm{hk}} \leq-q_{-}\right], \mathrm{P}_{+} \equiv \operatorname{Pr}\left[Q_{\mathcal{T}_{\mathrm{FP}}}^{\mathrm{hk}} \geq q_{+}\right] .
$$

The expression 29 can thus be written as

$$
\mathrm{P}_{-}\left\langle e^{\beta Q_{\mathcal{T}_{\mathrm{FP}} \mathrm{hk}}^{\mathrm{hk}}}\right\rangle_{-}+\mathrm{P}_{+}\left\langle e^{\beta Q_{\mathcal{F}_{\mathrm{FP}}}^{\mathrm{hk}}}\right\rangle_{+}=1,
$$


where $\left\langle e^{\beta Q_{\tau_{\mathrm{FP}}}^{\mathrm{hk}}}\right\rangle_{-}$and $\left\langle e^{\beta Q_{\mathcal{T}_{\mathrm{FP}}}^{\mathrm{hk}}}\right\rangle_{+}$are the conditional expectations of $e^{\beta Q_{\mathcal{T}_{\mathrm{FP}}}^{\mathrm{hk}}}$ given that $Q_{\mathcal{T}_{\mathrm{FP}}}^{\mathrm{hk}} \leq-q_{-}$or $Q_{\mathcal{T}_{\mathrm{FP}}}^{\mathrm{hk}} \geq q_{+}$, respectively. Solving (30) and (32) gives

$$
\begin{aligned}
& \mathrm{P}_{-}=\frac{\left\langle e^{\beta Q_{\mathrm{FF}_{\mathrm{FP}}}^{\mathrm{hk}}}\right\rangle_{+}-1}{\left\langle e^{\beta Q_{\mathcal{T}_{\mathrm{FP}}}^{\mathrm{hk}}}\right\rangle_{+}-\left\langle e^{\beta Q_{\mathcal{T}_{\mathrm{FP}}}^{\mathrm{hk}}}\right\rangle_{-}}, \\
& \mathrm{P}_{+}=\frac{1-\left\langle e^{\left.\beta Q_{\mathcal{T}_{\mathrm{FP}}}^{\mathrm{hk}}\right\rangle_{-}}\right.}{\left\langle e^{\beta Q_{\mathcal{T}_{\mathrm{FP}}}^{\mathrm{hk}}}\right\rangle_{+}-\left\langle e^{\beta Q_{\mathcal{T}_{\mathrm{FP}}}^{\mathrm{hk}}}\right\rangle_{-}} .
\end{aligned}
$$

If the process $Q_{t}^{\mathrm{hk}}$ is continuous, then $Q_{\mathcal{T}_{\mathrm{FP}}}^{\mathrm{hk}}=-q_{-}$or $Q_{\mathcal{T}_{\mathrm{FP}}}^{\mathrm{hk}}=q_{+}$, and we find exact expressions for the housekeeping heat splitting probabilities $\mathrm{P}_{-}$and $\mathrm{P}_{+}$:

$$
\mathrm{P}_{-}=\frac{e^{\beta q_{+}}-1}{e^{\beta q_{+}}-e^{-\beta q_{-}}}, \quad \mathrm{P}_{+}=\frac{1-e^{-\beta q_{-}}}{e^{\beta q_{+}}-e^{-\beta q_{-}}} .
$$

Note that the expressions (35) hold for the Itô diffusions (9). Consider now the maximum value of the housekeeping heat:

$$
Q_{\max }^{\mathrm{hk}} \equiv \max _{t \geq 0} Q_{t}^{\mathrm{hk}},
$$

i.e., the maximum amount of heat absorbed by the system, which characterizes the largest fluctuation that violates the average tendency $\left\langle Q_{t}^{\mathrm{hk}}\right\rangle \leq 0$ to dissipate. The cumulative distribution of $Q_{\max }^{\mathrm{hk}}$ is related to $\mathrm{P}_{+}$by

$$
\operatorname{Pr}\left[Q_{\max }^{\mathrm{hk}} \geq q_{+}\right]=\lim _{q_{-} \rightarrow \infty} \mathrm{P}_{+}=e^{-\beta q_{+}},
$$

with $q_{+} \geq 0$. Hence, the probability density of $Q_{\max }^{\mathrm{hk}}$ is an exponential distribution with mean value

$$
\left\langle Q_{\max }^{\mathrm{hk}}\right\rangle=k_{B} T .
$$

If we consider the maximum value of $Q_{\mathrm{hk}}$ at a finite time:

$$
Q_{\max , t}^{\mathrm{hk}} \equiv \max _{\tau \in[0, t]} Q_{\tau}^{\mathrm{hk}},
$$

then

$$
\operatorname{Pr}\left[Q_{\max , t}^{\mathrm{hk}} \geq q_{+}\right] \leq e^{-\beta q_{+}},
$$

and

$$
\left\langle Q_{\max , t}^{\mathrm{hk}}\right\rangle \leq k_{B} T,
$$

since $Q_{\max , \tau}^{\mathrm{hk}} \leq Q_{\max }^{\mathrm{hk}}$.

Although the relations (35 40 hold for continuous stochastic processes that do not satisfy detailed balance, $e^{\beta Q_{t}^{\text {hk }}}$ is also a martingale for processes that may have jumps. We therefore ask: what does the martingality of $e^{\beta Q_{t}^{\text {hk }}}$ imply for right-continuous stochastic processes, such as Markov jump processes?

For right-continuous stochastic processes that do not satisfy detailed balance, the relations (33) and (34) still hold. We use in them

$$
\left\langle e^{\beta Q_{\mathcal{T}_{\mathrm{FP}}}^{\mathrm{hk}}}\right\rangle_{-}=e^{-\beta q_{-}}\left\langle e^{-\beta \Delta_{-}}\right\rangle_{-},\left\langle e^{\beta Q_{\mathcal{T}_{\mathrm{FP}} \mathrm{h}}^{\mathrm{h}}}\right\rangle_{+}=e^{\beta q_{+}}\left\langle e^{\beta \Delta_{+}}\right\rangle_{+},
$$

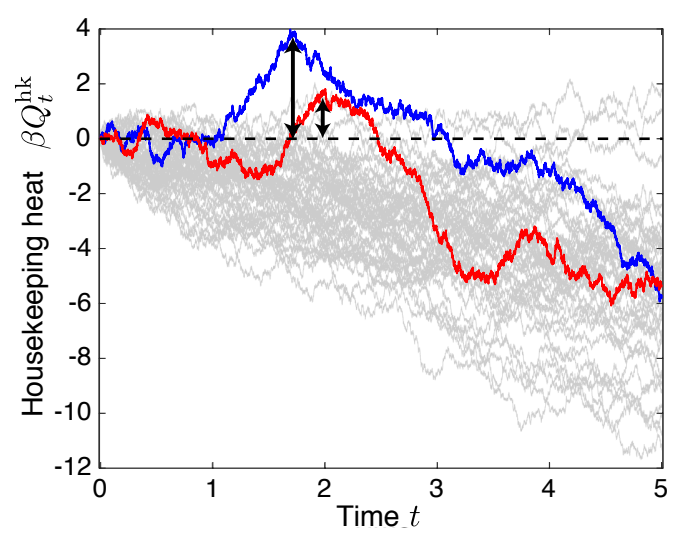

Fig. 1: Trajectories of the fluctuating housekeeping heat $Q_{t}^{\mathrm{hk}}$ (grey lines). The maximum value up to $t=5, Q_{\max , 5}^{\mathrm{hk}}$, of two highlighted trajectories of the housekeeping heat (red and blue lines) are indicated with double arrows. The time series are obtained from numerical simulations of the model 45, with $D=\omega=\beta=r=1.0$ with initial distribution $\rho_{0}(\theta)=\delta(\theta)$.

where $\Delta_{-}$and $\Delta_{+}$are the overshoot variables defined by $-q_{-}-Q_{\mathcal{T}_{\mathrm{FP}}}^{\mathrm{hk}}$ and $Q_{\mathcal{T}_{\mathrm{FP}}}^{\mathrm{hk}}-q_{+}$at the negative and positive threshold, respectively. Both $\Delta_{-}$and $\Delta_{+}$are positive random variables, and for continuous processes they are equal to zero. We see that in the limit of $q_{-} \rightarrow \infty$, $\left\langle e^{\beta Q_{\mathcal{T}_{\mathrm{FP}}}^{\mathrm{hk}}}\right\rangle_{-} \rightarrow 0$, and therefore,

$\operatorname{Pr}\left[Q_{\mathrm{sup}}^{\mathrm{hk}} \geq q_{+}\right]=\lim _{q_{-} \rightarrow \infty} \mathrm{P}_{+}=e^{-\beta q_{+}} /\left\langle e^{\beta \Delta_{+}}\right\rangle_{+} \leq e^{-\beta q_{+}}$.

The last inequality follows from $\Delta_{+}>0$ and thus $\left\langle e^{\beta \Delta_{+}}\right\rangle_{+}>1$. For the average, we have

$$
\left\langle Q_{\mathrm{sup}}^{\mathrm{hk}}\right\rangle \leq k_{B} T .
$$

Note that now we have used supremum value $Q_{\text {sup }}^{\text {hk }}$ instead of the maximum value $Q_{\max }^{\mathrm{hk}}$, since the maximum value may not exist for processes that may have jumps. The tightness of the bounds (43 44 depend on the magnitude of the overshoot variable $\left\langle e^{\beta \Delta_{+}}\right\rangle_{+}$, which is process dependent. For continuous processes, $\Delta_{+}=0$, and therefore the statistics of the long-time supremum are universal.

The martingality of $e^{\beta Q_{t}^{\mathrm{hk}}}$ implies that several fluctuation properties of the housekeeping heat of continuous processes that do no satisfy detailed balance are universal. These universal properties characterize fluctuations that oppose the general tendency of the process to dissipate. We have also found bounds that hold for any isothermal Markovian nonequilibrium process. These bounds are stronger than what we would expect from the integral fluctuation relation. In the following, we illustrate the bound (5) for the cumulative distribution of the finitetime maximum value of the housekeeping heat over a finite time.

Simulations. - We now test our results for the statistics of the maximum of the housekeeping heat with numerical simulations of a model described by the following 

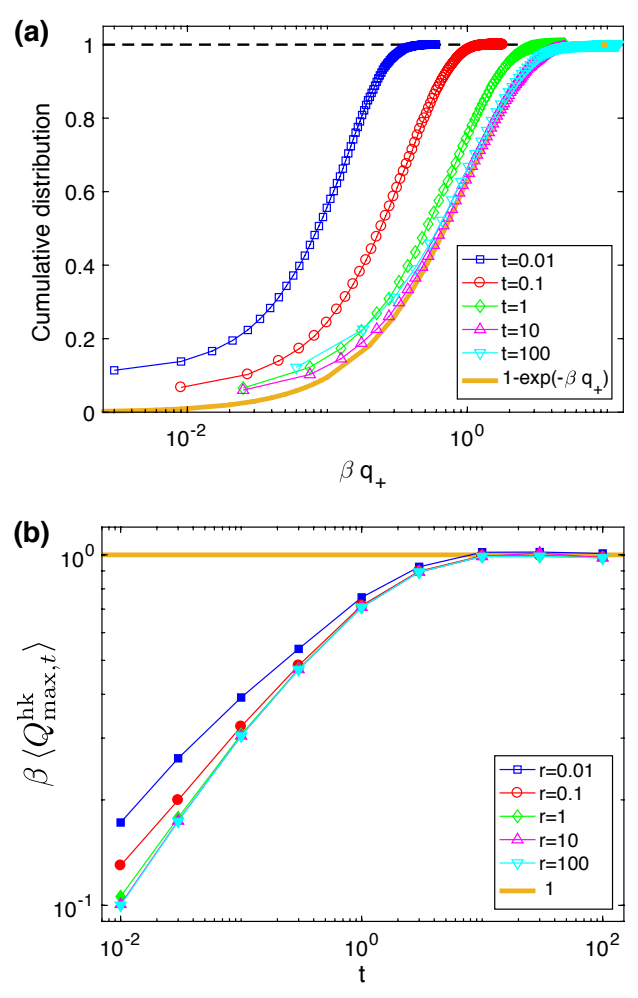

Fig. 2: Extreme-value statistics of the housekeeping heat. (a) Cumulative distribution of the maximum value (symbols with lines) of the housekeeping heat $Q_{\max , t}^{\mathrm{hk}}$ up to time $t$, for $r=$ 1 and different values of $t$ (legend). The yellow line is the theoretical bound (40) and the black dashed line is a guide to the eye. (b) Average value $\left\langle Q_{\max , t}^{\mathrm{hk}}\right\rangle$ in units of $k_{\mathrm{B}} T$ as a function of $t$ for different values of the driving speed $r$ (legend). The yellow line is set to unity, corresponding to the bound (41). Here, we have taken $D=1, \omega=1,10^{4}$ independent realizations of the dynamics with initial distribution $\rho_{0}(\theta)=\delta(\theta)$, and integration time step $\Delta t=10^{-4}$.

one-dimensional overdamped Langevin equation

$$
\frac{\mathrm{d} \theta_{t}}{\mathrm{~d} t}=\omega-\partial_{\theta} V\left(\theta_{t} ; t\right)+\sqrt{2 D} \eta_{t} .
$$

Here $\eta_{t}$ is a Gaussian white noise satisfying $\left\langle\eta_{t}\right\rangle=$ $0,\left\langle\eta_{t} \eta_{t^{\prime}}\right\rangle=\delta\left(t-t^{\prime}\right), D=1 / \beta$ due to the fluctuationdissipation relation (here we take unity for the friction constant). The system is driven out of equilibrium by a constant external torque $\omega$ and a time-dependent potential

$$
V(\theta ; t)=(1 / \beta) \ln \left(\cos \theta+\lambda_{t}\right) ; \lambda_{t}=2+r t,
$$

where $r>0$ is the speed of the time-dependent protocol $\lambda_{t}$. The corresponding accompanying density is

$$
\pi_{t}(\theta)=\mathcal{N}^{-1} e^{-\beta U(\theta)} \int_{\theta}^{\theta+2 \pi} \mathrm{d} \theta^{\prime} e^{\beta U\left(\theta^{\prime}\right)},
$$

where $U(\theta) \equiv-\omega \theta+V(\theta)$, and $\mathcal{N}$ is a normalization constant 35]. Using (47) in (13), we derive the following analytical expression for the housekeeping heat:

$$
\beta Q_{t}^{\mathrm{hk}}=-\int_{0}^{t} \frac{2 \lambda_{s}+2 \cos \theta_{s}}{2 \lambda_{s}-\sin \theta_{s}-\cos \theta_{s}} \circ \mathrm{d} \theta_{s} .
$$

We perform numerical simulations of Eq. 45 using Heun's numerical integration scheme. his numerical scheme is shown to converge to the solution of Langevin equations of the type (45) with the force interpreted in the Stratonovich sense [36], as required to minimize numerical errors in stochastic energetics (see Sec. 4.1.2.5 in 37]). From dynamical trajectories, we evaluate the housekeeping heat using (48). Trajectories of the fluctuating housekeeping heat exhibit positive excursions corresponding to transient absorptions of housekeeping heat while on average tend to decrease in time (see Fig. 1). From these trajectories, we then compute empirical cumulative distributions of the finite-time maximum of the housekeeping heat $Q_{t}^{\text {hk }}$ for a given value of $r$ (see Fig. 2 a). The empirical distributions obey the inequality $\operatorname{Pr}\left[Q_{\max , t}^{\mathrm{hk}} \leq q_{+}\right] \geq$ $1-e^{-\beta q_{+}}$for both small and large values of $t$. Interestingly, the inequality becomes tighter when $t$ is large, in agreement with (37). Furthermore, we measure the average finite-time maximum of the housekeeping heat $\left\langle Q_{\max , t}^{\mathrm{hk}}\right\rangle$ for different values of the driving speed $r$ and the observation time $t$. Our numerical results fulfil the inequality $\beta\left\langle Q_{\max , t}^{\mathrm{hk}}\right\rangle \leq 1$ for all the tested parameter values, and the bound is tighter when $t$ is large, in agreement with our theory.

Discussion. - Our work demonstrates the power of martingale theory to describe extreme-value and stopping statistics of the fluctuating housekeeping heat in nonequilibrium processes. Our results can be extended also to non-isothermal classical systems, for which $e^{-S_{t}^{\mathrm{a}} / k_{\mathrm{B}}}$ is a path-probability ratio 19,20$]$ and thus a martingale, i.e. $\left\langle e^{-S_{t}^{\mathrm{a}} / k_{\mathrm{B}}} \mid X_{[0, \tau]}\right\rangle=e^{-S_{\tau}^{\mathrm{a}} / k_{\mathrm{B}}}$ for $t \geq \tau$. Here $S_{t}^{\mathrm{a}}$ is the adiabatic entropy production i.e. the fluctuating entropy production required to keep the system out of equilibrium when the system is driven quasi-statically. Interestingly, $S_{t}^{\mathrm{a}}$ follows the same fluctuation properties as $-Q_{t}^{\mathrm{hk}} / T$ concerning extrema and stopping-time statistics. We envisage that our theory could be tested experimentally with small colloidal, biological, electronic systems 3843 and that our results could also be extended to e.g. classical systems with long-range interactions 44,45 and quantum systems 46.

Acknowledgments.- The authors thank MPIPKS, Dresden for hosting them during the workshop "Stochastic Thermodynamics: Experiment and Theory" in September 2018 when major part of this work was done. We acknowledge fruitful discussions with Simone Pigolotti and Frank Jülicher. 


\section{REFERENCES}

[1] J. Ville, Etude critique de la notion de collectif (PhD Thesis) Gauthier-Villars, Paris (1939).

[2] J. L. Doob, Stochastic processes (Wiley, New York, 1953).

[3] D. Williams, Probability with martingales (Cambridge University Press, Cambridge, 1991).

[4] R. S. Liptser and A. N. Shiryaev, Statistics of random processes: I \& II (Springer Science \& Business Media, Berlin, 2013).

[5] B. Øksendal, Stochastic differential equations (Springer, Berlin, 2003).

[6] A sufficient condition for $I_{t}=\int_{0}^{t} F\left(X_{s}\right) \cdot d \mathcal{W}_{s}$ to be a martingale is $\left\langle\int_{0}^{t} F^{2}\left(X_{s}\right) d s\right\rangle<\infty$ for all $t \geq 0$.

[7] Probability ratios of densities of trajectories are in fact Radon-Nikodym derivatives between two mutually absolute continuous measures.

[8] S. Pliska, Introduction to mathematical finance (Blackwell publishers, Oxford, 1997).

[9] I. Neri, É. Roldán and F. Jülicher, Phys. Rev. X 7, 011019 (2017).

[10] R. Chetrite and S. Gupta, J. Stat. Phys. 143, 543 (2011).

[11] B. Ventéjou and K. Sekimoto, Phys. Rev. E 97, 062150 (2018).

[12] M. Bauer and D. Bernard, Phys. Rev. A 84, 044103 (2011)

[13] S. L. Adler, D. C. Brody, T. A, Brun and L. F. Hughston, J. Phys. 34, 8795 (2001).

[14] S. Singh et al., arXiv:1712.01693 (2017).

[15] Y. Oono and M. Paniconi, Prog. Theor. Phys. Suppl. 130, 29 (1998)

[16] K. Sekimoto, Prog. Theor. Phys. Suppl. 130, 17 (1998).

[17] T. Hatano and S.-i. Sasa, Phys. Rev. Lett. 86, 3463 (2001).

[18] T. Speck and U. Seifert, J. Phys. A, 38, L581 (2005).

[19] M. Esposito and C. Van den Broeck, Phys. Rev. E, 82, 011143 (2010).

[20] C. Van den Broeck and M. Esposito, Phys. Rev. E 82, 011144 (2010).

[21] A bounded stopping time $\mathcal{T} \in[0, \tau]$ with $\tau$ a constant fixed time.

[22] P. Hänggi, Helv. Phys. Acta 53, 491 (1980).

[23] Y. L. Klimontovich, Phys.-Usp. 37, 737 (1994).

[24] Y. L. Klimontovich, Phys. A 163, 515 (1990).

[25] D. L. Fisk, Quasi-martingales and stochastic integrals, Tech Rep 1 Dept Math Univ Michigan State (1963).

[26] R. L. Stratonovich, Conditional Markov process and their applications to the theory of optimal control (Amer. Elsiever, New York, 1968).

[27] P. Hänggi, H. Thomas, Phys. Rep. 88, 207 (1982).

[28] R. J. Harris and G. M. Schütz, J. Stat. Mech. 2007(07), P07020 (2007).

[29] R. Chetrite and K. Gawedzki, Comm. Math. Phys. 282(2), 469 (2008).

[30] R. García-García, D. Domínguez, V. Lecomte and A. B. Kolton, Phys. Rev. E 82, 030104(R) (2010).

[31] M. Esposito, U. Harbola and S. Mukamel, Phys. Rev. E 76, 031132 (2007).

[32] S. Pigolotti, I. Neri, É. Roldán and F. Jülicher, Phys. Rev. Lett. 119, 140604 (2017).

[33] S. L. Heston, Rev. Financial Stud. 6(2), 327-343 (1993).

[34] H.-M. Chun and J. D. Noh, arXiv:1810.01121 (2018).
[35] P. Reimann, Phys. Rep., 361, 57 (2002).

[36] W. Rümelin, SIAM J. Num. Anal., 19(3), 604-613 (1982).

[37] K. Sekimoto, Stochastic Energetics (Springer, Berlin, 2010).

[38] E. H. Trepagnier, C. Jarzynski, F. Ritort, G. E. Crooks, C. J. Bustamante, and J. Liphardt, PNAS, 101(42), 15038-15041 (2004).

[39] S. Toyabe, T. Watanabe-Nakayama, T. Okamoto, S. Kudo, and E. Muneyuki, PNAS, 108(44), 17951-17956 (2011).

[40] Y. Jun, M. Gavrilov, and J. Bechhoefer, Phys. Rev. Lett., 113(19), 190601 (2014).

[41] I. A. Martínez, É. Roldán, L. Dinis, and R. A. Rica, Soft Matter, 13(1), 22-36 (2017).

[42] S. Ciliberto, Phys. Rev. X, 7(2), 021051 (2017).

[43] A. Ronzani, B. Karimi, J. Senior, Y.-C. Chang, J. T. Peltonen, C. Chen and J. P. Pekola, Nature Phys. 14, 991-995 (2018).

[44] S. Gupta, T. Dauxois and S. Ruffo, EPL 113, 60008 (2016).

[45] S. Gupta, A. Campa and S. Ruffo, Statistical Physics of Synchronization (Springer, Berlin, 2018).

[46] G. Manzano, J. M. Horowitz, and J. M. R. Parrondo, Phys. Rev. X, 8(3), 031037 (2018).

Appendix A: Proof of Eq. 24). - The Ito expression for the housekeeping heat [cf. Eq. 223] ] can be expressed in terms of the effective current $\overrightarrow{J_{t}^{\pi}}=\vec{F}_{t} \pi_{t}-\mathbf{D}_{t}$. $\vec{\nabla} \pi_{t}$ as

$$
\begin{aligned}
Q_{t}^{\mathrm{hk}} & =-k_{\mathrm{B}} T \int_{0}^{t} \frac{\mathbf{D}_{s}^{-1} \vec{J}_{s}^{\pi}}{\pi_{s}}\left(\vec{X}_{s}\right) \cdot \mathrm{d} \vec{X}_{s} \\
& -k_{\mathrm{B}} T \int_{0}^{t} \mathrm{~d} s\left[\mathbf{D}_{s} \cdot \vec{\nabla} \cdot\left(\frac{\mathbf{D}_{s}^{-1} \vec{J}_{s}^{\pi}}{\pi_{s}}\right)\right]\left(\vec{X}_{s}\right) .
\end{aligned}
$$

Using Ito's lemma in the above equation gives

$$
\begin{aligned}
\mathrm{d} e^{\beta Q_{t}^{\mathrm{hk}}} & =-e^{\beta Q_{t}^{\mathrm{hk}}}\left[\frac{\mathbf{D}_{t}^{-1} \vec{J}_{t}^{\pi}}{\pi_{t}}\right]\left(\vec{X}_{t}\right) \cdot \mathrm{d} \vec{X}_{t} \\
& -e^{\beta Q_{t}^{\mathrm{hk}}}\left[\mathbf{D}_{t} \cdot \vec{\nabla} \cdot\left(\frac{\mathbf{D}_{t}^{-1} \vec{J}_{t}^{\pi}}{\pi_{t}}\right)\right]\left(\vec{X}_{t}\right) \mathrm{d} t \\
& +\frac{1}{2} e^{\beta Q_{t}^{\mathrm{hk}}}\left[\frac{\mathbf{D}_{t}^{-1} \vec{J}_{t}^{\pi}}{\pi_{t}}\right] \cdot 2 \mathbf{D}_{t} \cdot\left[\frac{\mathbf{D}_{t}^{-1} \vec{J}_{t}^{\pi}}{\pi_{t}}\right]\left(\vec{X}_{t}\right) \mathrm{d} t .
\end{aligned}
$$

Substituting Eq. (9) of the Main text in the first line of 50), using the properties $\vec{\nabla} \cdot \vec{J}_{t}^{\pi}=0$ (which is a consequence of the definition (12) of the accompanying distribution) and the mathematical property $\nabla_{\mu}\left(\mathbf{D}^{-1}\right)=$ $-\mathbf{D}^{-1} \cdot\left(\nabla_{\mu} \mathbf{D}\right) \cdot \mathbf{D}^{-1}-$ with $\nabla_{\mu}$ given by the $\mu$ component of $\vec{\nabla}-$, we obtain after some algebra

$$
\frac{\mathrm{d}}{\mathrm{d} t} e^{\beta Q_{t}^{\mathrm{hk}}}=-\sqrt{2} e^{\beta Q_{t}^{\mathrm{hk}}}\left[\frac{\vec{J}_{t}^{\pi} \cdot \mathbf{D}_{t}^{-1 / 2}}{\pi_{t}}\right]\left(\vec{X}_{t}\right) \cdot \vec{\xi}_{t} .
$$

